\title{
Atomistic simulation of damage accumulation and amorphization in $\mathrm{Ge}$
}

Jose L. Gomez-Selles, Alain Claverie, Benoit Sklenard, Francis Benistant, and Ignacio Martin-Bragado

Citation: Journal of Applied Physics 117, 055703 (2015); doi: 10.1063/1.4907211

View online: https://doi.org/10.1063/1.4907211

View Table of Contents: http://aip.scitation.org/toc/jap/117/5

Published by the American Institute of Physics

\section{Articles you may be interested in}

Retraction: "Atomistic simulation of damage accumulation and amorphization in Ge" [J. Appl. Phys. 117, 055703 (2015)]

Journal of Applied Physics 121, 049901 (2017); 10.1063/1.4974919

Retraction: "Bulk- and layer-heterojunction phototransistors based on poly[2-methoxy-5-(2'-ethylhexyloxy-p-

phenylenevinylene)] and PbS quantum dot hybrids" [Appl. Phys. Lett. 106, 253501 (2015)]

Applied Physics Letters 110, 089901 (2017); 10.1063/1.4975640

Bulk- and layer-heterojunction phototransistors based on poly [2-methoxy-5-(2'-ethylhexyloxy-p-

phenylenevinylene)] and $\mathrm{PbS}$ quantum dot hybrids

Applied Physics Letters 106, 253501 (2015); 10.1063/1.4922917

In referees we trust?

Physics Today 70, 44 (2017); 10.1063/PT.3.3463

Retraction: "Experimental techniques for imaging and measuring transient vapor nanobubbles" [Appl. Phys. Lett. 101, $264102(2012)]$

Applied Physics Letters 110, 129901 (2017); 10.1063/1.4978413

Wealth inequality: The physics basis

Journal of Applied Physics 121, 124903 (2017); 10.1063/1.4977962

\section{Scilight}

Sharp, quick summaries illuminating the latest physics research

\section{Sign up for FREE!}

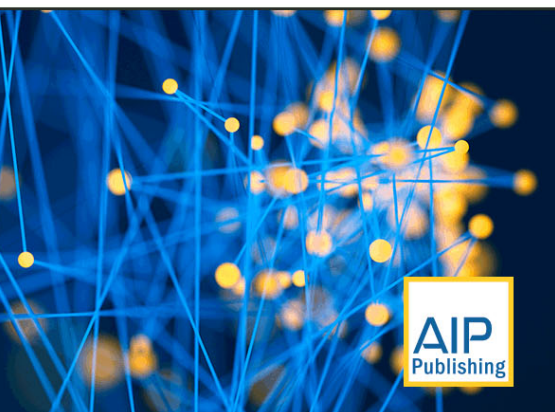




\title{
Atomistic simulation of damage accumulation and amorphization in $\mathrm{Ge}$
}

\author{
Jose L. Gomez-Selles, ${ }^{1, a)}$ Alain Claverie, ${ }^{2}$ Benoit Sklenard, ${ }^{3}$ Francis Benistant, ${ }^{4}$ \\ and Ignacio Martin-Bragado ${ }^{1}$ \\ ${ }^{1}$ IMDEA Materials Institute, Eric Kandel 2, 28906 Getafe, Madrid, Spain \\ ${ }^{2}$ CEMES/CNRS, 29 rue J. Marvig, 31055 Toulouse Cedex, France \\ ${ }^{3}$ CEA, LETI, 17 rue des Martyrs, 38054 Grenoble Cedex 9, France \\ ${ }^{4}$ GLOBALFOUNDRIES Singapore Pte Ltd., 60 Woodlands Industrial Park D Street 2, Singapore 738406 , \\ Singapore
}

(Received 31 July 2014; accepted 20 January 2015; published online 3 February 2015)

\begin{abstract}
Damage accumulation and amorphization mechanisms by means of ion implantation in Ge are studied using Kinetic Monte Carlo and Binary Collision Approximation techniques. Such mechanisms are investigated through different stages of damage accumulation taking place in the implantation process: from point defect generation and cluster formation up to full amorphization of Ge layers. We propose a damage concentration amorphization threshold for Ge of $\sim 1.3 \times 10^{22} \mathrm{~cm}^{-3}$ which is independent on the implantation conditions. Recombination energy barriers depending on amorphous pocket sizes are provided. This leads to an explanation of the reported distinct behavior of the damage generated by different ions. We have also observed that the dissolution of clusters plays an important role for relatively high temperatures and fluences. The model is able to explain and predict different damage generation regimes, amount of generated damage, and extension of amorphous layers in Ge for different ions and implantation conditions. (C) 2015 AIP Publishing LLC. [http://dx.doi.org/10.1063/1.4907211]
\end{abstract}

\section{INTRODUCTION}

Ge is a promising high mobility channel alternative material for the future generation of pMOS transistors, ${ }^{1-3}$ and a serious effort has been invested in the last years in order to understand the mechanisms of Ge damage accumulation and amorphization caused by ion implantation. ${ }^{4-10} \mathrm{~B}$-doping in $\mathrm{Ge}$ is of particular interest due to its low interstitial $(I)$ mediated diffusion. ${ }^{11}$ In this context of Ge implementation in ultimate microelectronic devices, both bulk $\mathrm{Ge}$ and $\mathrm{Ge}$ on insulator $(\mathrm{GeOI})$ wafers are treated with ion-beam techniques in which damage accumulation and amorphization play a significant role. ${ }^{12}$ Thus, it becomes important to understand amorphization processes in order to predict and optimize the position and extension of amorphous layers in all relevant implantation conditions.

Within this framework, the Critical Damage Energy Density (CDED) model $^{13}$ was able to accurately reproduce amorphous/crystalline $(\alpha / c)$ interface locations for relatively heavy ions and wide fluence ranges. ${ }^{7}$ Nevertheless, predictions for low mass ions, such as B, could not be provided. Despite other models also reported consider the competition between damage buildup and its dynamic recovery, ${ }^{5}$ a deeper understanding of the energetics of recombination and migration barriers was also suggested to be needed.

In this work, we introduce a comprehensive atomistic model of damage accumulation and amorphization by means of ion implantation in Ge. Explanations for the differences between the damage caused by ions of different masses are presented and validated by reproducing experimental observations of $\mathrm{B}, \mathrm{F}, \mathrm{Ga}$, and $\mathrm{Ge}$ implants. This is achieved

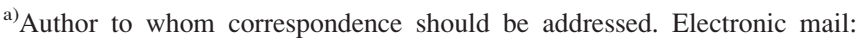
joseluis.gomezselles@imdea.org
}

through an intensive study of the energetics of recombination of "amorphous" pockets $(A P)$ coupled with a realistic calculation of the damage distribution within the volume of implantation. Damage accumulation mechanisms towards amorphization have been examined. Pure collisional stages are separated from dynamic annealing effects, providing clarifications on different steps of $\mathrm{Ge}$ amorphization. Validity of the model is shown by simulating own experiments and others reported in the literature. ${ }^{4,5,7-9,14}$

\section{MODEL}

By coupling a Binary Collision Approximation (BCA) code with a physically based Kinetic Monte Carlo (KMC) method, a complex variety of mechanisms induced by ion implantation can be simulated. ${ }^{15-17}$ Among BCA codes, SRIM $^{18}$ is widely used although it does not consider the crystallinity of the target material. This might lead to inaccurate damage or doping profile predictions, and the models used need to be chosen attending to specific experiments. ${ }^{19}$ Particularly, light ions are easily affected by channeling effects. ${ }^{20,21}$ To take this into account, the Sentaurus Process BCA module ${ }^{22}$ was used to generate the initial damage (Is and vacancies, $V \mathrm{~s}$ ) distribution. The threshold displacement energy per atom, $E_{t}$, used in this model is $30 \mathrm{eV} / \mathrm{atom}^{20,23}$

It is widely accepted that damage cascade generation occurs at a different time scale than the dynamic events taking place during the implantation process. ${ }^{24}$ As a consequence, damage due to a single ion can be calculated, and then, its dynamic evolution computed. ${ }^{25}$ In this model, damage cascades taken from BCA calculations are introduced at the desired rate into the Object-KMC simulator for damage irradiation evolution and defect diffusion, $\mathrm{MMonCa},{ }^{26,27}$ in order to simulate dynamic annealing effects. 
Three different damage generation regimes have been reported for ion implantation in both $\mathrm{Ge}$ and $\mathrm{Si}$ materials (see Refs. 6 and 28, respectively, and references therein), but these regimes have not been as studied in $\mathrm{Ge}$ as in $\mathrm{Si}$. We model these implantation regimes as follows. Damage is produced in the simulated target at the crystalline regime (i.e., calculated with channeling effects), and its production grows slowly (Region I) until it locally overcomes a certain threshold of damage concentration. At this point, areas of $c$-Ge relax towards the $\alpha$-Ge phase and, as in $\mathrm{Si}^{28}$ the mixture of these different phases result in a faster damage growth regime (Region II). These areas nucleate until a full layer is amorphized and, as the lattice has relaxed, no more damage can be generated. In this new regime (Region III), only cascades computed without channeling effects are used and the induced damage only contributes to the widening of the layer.

Regarding identification of amorphous layers, it is a complex task to choose a consistent criterion. For instance, different models have been reported with different standards for Si amorphization modeling. ${ }^{29,30}$ In this model, we identify the local position of the transition between the two phases, and place the $\alpha / c$ interface in the mean value of these local interfaces. This also provides a value of the simulated roughness of such interface, resulting from regions where both phases coexist.

Dynamic annealing events are modeled through recombination energies which depend on the damage configuration. Agglomeration of $I \mathrm{~s}$ and $V \mathrm{~s}$ gives rise to compact damaged Ge regions of different sizes, namely, $A P$ s. The effective size, $s$, of an $A P$ formed by $n I$ s and $m V \mathrm{~s}$ is computed as $s=\min (n, m)$. This definition accounts for the number of available $I V$-pairs inside an $A P$ able to perform the reaction $I+V \rightarrow 0 .^{28}$ For every recombination event, an $A P$ of size $s$ shrinks to $(s-1)$ at a rate of ${ }^{17}$

$$
\nu=\alpha s^{\beta} \exp \left[-E_{\text {act }}(s) / k T\right],
$$

where $\alpha=5 \times 10^{-3} \mathrm{~cm}^{2} \mathrm{~s}^{-1}, \beta=1$ and $E_{a c t}(s)$ is the activation energy of Ge $A P$ recombination shown in Fig. 1 (solid line). One immediate result of both this effective size definition

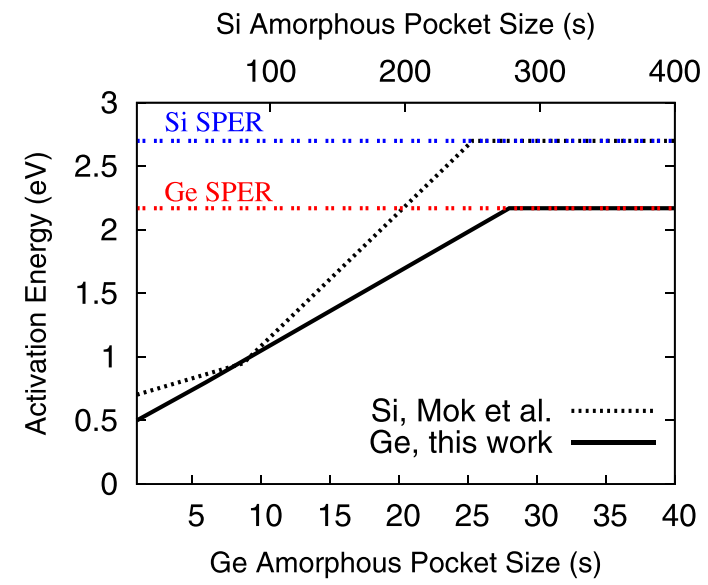

FIG. 1. Activation energies of recombination as a function of the Amorphous Pocket effective size, $s$, for $\mathrm{Si}^{17}$ (dashed line) and $\mathrm{Ge}$, this work (solid line). SPER energies are also shown for $\mathrm{Si}^{35,36}$ (blue) and $\mathrm{Ge}^{37,38}$ (red). and the proposed size dependency is that stability of these clusters may be thought as a "coordination" between the number of $I \mathrm{~s}$ and $V \mathrm{~s}$. This means that an $I_{1} V_{9}(s=1)$ would recombine much easier than an $I_{5} V_{5}(s=5)$, even though both are composed by the same number of defects. In Fig. 1, energies reported for $A P$ recombination in $\mathrm{Si}^{17}$ are also presented. Further discussions on these energies and comparisons between both materials are given in Sec. III B.

Prefactors and energies for the Arrhenius expressions of migration and formation of $I \mathrm{~s}$ and $V \mathrm{~s}$ have been calibrated in agreement with previous studies, ${ }^{31-34}$ and are shown in Table I.

A comprehensive discussion on the formation and binding energies of pure $I$ - and $V$-type clusters is out of the scope of this work. Nevertheless, a simple calibration has been performed in order to be able to reproduce some experimental results. This calibration is discussed at the end of Sec. III.

\section{RESULTS AND DISCUSSION}

Damage accumulation by means of ion implantation, amorphizing or not, is a highly complex process in which several mechanisms take place. Thus, results are presented by separating the different stages of Ge amorphization. In Sec. III A, amorphization is discussed by analyzing the mechanisms taking place when dynamic annealing events can be neglected. In Sec. III B, dynamic annealing effects of defect recombination are studied. Section III C is focused on differences reported between ions, and their effects on damage generation and amorphization. Finally, in Sec. IIID, mechanisms of damage dissolution are discussed.

Despite these results are separated in such way, all simulations have been performed using the same model and calibration.

\section{A. Amorphization threshold}

A $\langle 100\rangle$ germanium wafer with a resistivity in the $15-22 \Omega$ range was implanted with $40 \mathrm{keV} 1 \times 10^{15} \mathrm{~B} / \mathrm{cm}^{2}$ ions at $100 \mathrm{~K}$. This temperature was chosen to minimize dynamic annealing events, ${ }^{10}$ focusing only on the pure collisional part of the damage buildup mechanisms towards amorphization. Then, the sample was prepared for transmission electron microscopy (TEM) according to the standard preparation procedure consisting of mechanical grinding and ion milling at the same temperature of implantation to minimize defect annealing. Cross sectional TEM (XTEM) observation of the sample at room temperature (RT) showed a continuous amorphous layer extending from the surface up to $\sim 135 \mathrm{~nm}$ (not shown). The temperature of analysis is not expected to produce any further impact in the position of the measured $\alpha / c$ interface position, once formed, since higher

TABLE I. Migration and formation energies $\left(E_{m}, E_{f}\right)$ and prefactors $\left(D_{0, m}\right.$, $\left.D_{0_{f}}\right)$ of $\mathrm{Ge} I \mathrm{~s}$ and $V \mathrm{~s}$ used in this model.

\begin{tabular}{lcccc}
\hline \hline & $D_{0, m}\left(\mathrm{~cm}^{2} \mathrm{~s}^{-1}\right)$ & $E_{m}(\mathrm{eV})$ & $D_{0, f}\left(\mathrm{~cm}^{-3}\right)$ & $E_{f}(\mathrm{eV})$ \\
\hline$I$ & $1 \times 10^{-5}$ & 0.8 & $1 \times 10^{28}$ & 3.75 \\
$V$ & 10 & 0.77 & $1 \times 10^{24}$ & 2.43 \\
\hline \hline
\end{tabular}


temperatures would be needed to induce the Solid Phase Epitaxial Regrowth (SPER) process. ${ }^{9,37,38}$

Simulating such conditions, without defining any amorphization threshold, a damage concentration of $\sim 1.3 \times 10^{22} \mathrm{~cm}^{-3}$ was obtained at the mentioned depth. The use of this value as the amorphization threshold, i.e., $\rho_{t} \simeq 1.3 \times 10^{22} \mathrm{~cm}^{-3}$, gave rise to an $\alpha / c$ transition at $(140 \pm 10) \mathrm{nm}$, in good agreement with the observation. This value of $\rho_{t}$ is also able to reproduce the $\alpha / c$ locations measured by Impellizzeri et al. ${ }^{8}$ for $35 \mathrm{keV} \mathrm{B}$ implants at liquid nitrogen temperature $\left(\mathrm{LN}_{2} \mathrm{~T}\right)$. These results, obtained for cryogenic conditions, are presented among others, for different experimental conditions, later in this work in Fig. 6. It is important to notice that the presented value of $\rho_{t}$ does not depend on the implantation conditions.

In the mentioned work of Impellizzeri et al. ${ }^{8}$ channeling Rutherford backscattering spectrometry (c-RBS) was used to determine the amount of damage caused in the Ge lattice after implantations. Such measurements resulted in the quantity of damage per unit of implanted area, integrated over the whole depth of the sample. We have analyzed those results together with our simulations in order to better understand the amorphization process of Ge. Fig. 2 is a comparison between experimental ${ }^{8}$ (symbols) and simulated (lines) results of the displaced $\mathrm{Ge}$ atoms after $35 \mathrm{keV} \mathrm{B}$ implants at $\mathrm{LN}_{2} \mathrm{~T}$ for different fluences. The dashed line represents simulation results without settling a value of $\rho_{t}$. It is observed that the damage increases linearly without any limit, and no agreement could be found. These simulations do not either reproduce different implantation regimes. ${ }^{6}$ On the contrary, results obtained by applying the amorphization model (solid line) not only match a perfect agreement, but also helped to elucidate the fundamental mechanisms of Ge amorphization. As c-RBS measurements are able to extract the quantity of displaced Ge atoms (i.e., I atoms), a distinction between the three different damage generation regimes can be made (vertical dashed lines). Damage is accumulated linearly (Region I) until the amorphization threshold is locally exceeded, and that part of the lattice relaxes. This means that introducing $\sim 30 \%$ damage concentration of Ge atomic density, $\rho=4.42 \times 10^{22} \mathrm{~cm}^{-3}$, is enough to collapse the lattice structure of Ge. This results in a faster damage accumulation (Region II). At this point, no more damage can be generated

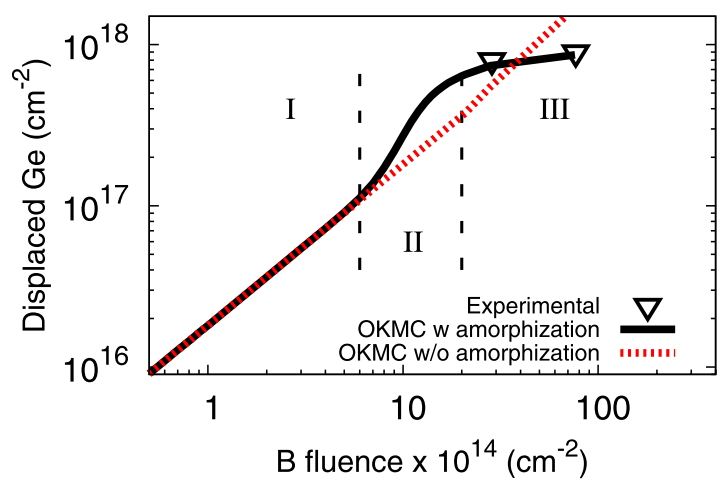

FIG. 2. Displaced Ge atoms for different $35 \mathrm{keV}$ B fluences at $\mathrm{LN}_{2} \mathrm{~T}$. Symbols represent experimental data. ${ }^{8}$ Simulation results with (solid line) and without (dashed line) the amorphization model are presented. Implantation regimes (I, II, and III) are separated by dashed vertical lines. into those amorphized areas, and the damage accumulation saturates at the Region III for enough extended amorphous layers. This interpretation explains the measured values by c-RBS, their tendency, and the observed implantation regimes. This behavior has already been widely studied in $\mathrm{Si}^{28}$ As we consider only a structural phase transition without dynamic effects, added to the fact that $\mathrm{Si}$ and Ge lattices are similar, the model can be thought to be applicable to both $\mathrm{Si}$ and Ge materials. Mentioned tendencies and results for c-RBS measurements are also observed for other conditions and compared with our results later in this work.

\section{B. Dynamic annealing}

Once the amorphization threshold was chosen, we considered dynamic events, which occur at higher temperatures, causing a competition between damage generation and its dynamic annealing. ${ }^{5}$ Such events are modeled through the $A P$ recombination energies already shown in Fig. 1 . Ge recombination energies start at $\sim 0.5 \mathrm{eV}$ for the smallest sizes and grow up to the activation energy of SPER of $2.17 \mathrm{eV}$ for a planar $\alpha / \mathrm{c}$ interface. ${ }^{37,38}$ This approach has already been validated for $\mathrm{Si}$ in both continuous and atomistic models. ${ }^{17,28,39}$

It is noticeable that $\mathrm{Ge}$ recombination energies presented in this work are generally lower than those reported for $\mathrm{Si}^{17}$ This is connected to the fact that, in Ge, damage recovery occurs at lower temperatures than in $\mathrm{Si}^{4}$ To better clarify this statement, and to evaluate the influence of different prefactors in Eq. (1), we have studied the differences between calculated total rates for two different temperatures. Fig. 3 is a comparison between $\mathrm{Si}^{17}$ and $\mathrm{Ge} A P$ recombination rates as a function of their effective size, $s$, at both $300 \mathrm{~K}$ and $500 \mathrm{~K}$. AP recombination rates are generally orders of magnitude lower in $\mathrm{Si}$ than in Ge. This means that for a given time, temperature and $A P$ distribution, much more recombination events will take place in $\mathrm{Ge}$ than in $\mathrm{Si}$. It is worth mentioning that for $\mathrm{Ge}$, these clusters of small sizes have been able to explain the fundamental processes of damage buildup, when comparing with the larger reported sizes in $\mathrm{Si}$.

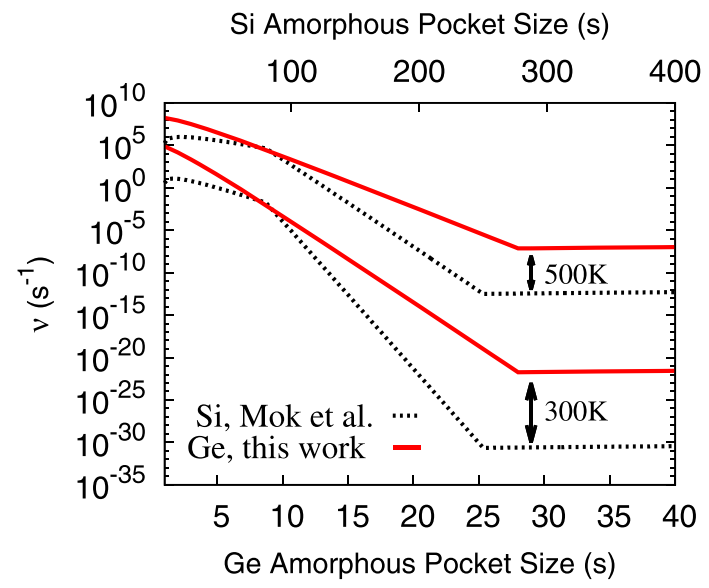

FIG. 3. $A P$ recombination rates, $\nu$, as a function of their effective size, $s$, for $\mathrm{Si}^{17}$ (dashed line) and $\mathrm{Ge}$, this work (solid line), for both $300 \mathrm{~K}$ and $500 \mathrm{~K}$. Corresponding temperatures are indicated with arrows between $\mathrm{Si}$ and $\mathrm{Ge}$ lines. 


\section{Different ions}

It has been reported that the damaged regions caused by $\mathrm{B}$ and other light ions are smaller and more diluted than by heavier ions. ${ }^{7,40}$ To study the possible variations between different ion masses, simulations of B and Ge implantations were performed. Fig. 4 shows the 2D concentration distribution histogram for $A P \mathrm{~s}$ resulting from simulations of $150 \mathrm{keV} \mathrm{Ge}$ (a) and $40 \mathrm{keV} \mathrm{B}$ (b) implants at $\mathrm{LN}_{2} \mathrm{~T}$. The color represents the percent contribution to the total damage of every $A P$, formed by $n I \mathrm{~s}$ (y-axes) and $m V \mathrm{~s}$ (x-axes). Fluences were chosen to generate $\sim 22000 I \mathrm{~s}$ and $V \mathrm{~s}$ at both conditions. The low temperature was again chosen to study the damage composition without further dynamic annealing effects. $A P$ s resulting from $\mathrm{Ge}$ implantations consist of higher number of $I \mathrm{~s}$ and $V \mathrm{~s}$ than those generated by B. In Fig. 4(c), the normalized cumulative damage is presented for those $\mathrm{Ge}$ (solid line) and B (dashed line) implants. While $\sim 90 \%$ of the total damage caused by B ions is constituted by $A P$ s formed by less than 5 defects, higher number of defects per $A P(\sim 20)$ are needed to reach the same contribution to the total damage for a $\mathrm{Ge}$ implant. This examination suggests that the damage caused by Ge ions is more compact than the one caused by B ions.

To evaluate the impact of the presented recombination rates in the damage distribution caused by both $\mathrm{B}$ and $\mathrm{Ge}$ ions, we have made a comparison of the simulated induced damage by $40 \mathrm{keV}, 5 \times 10^{14} \mathrm{~B} / \mathrm{cm}^{2}$ and $150 \mathrm{keV}, 2.25 \times 10^{13}$ $\mathrm{Ge} / \mathrm{cm}^{2}$ implants at both $\mathrm{LN}_{2} \mathrm{~T}$ and $0^{\circ} \mathrm{C}$, presented in Fig. 5 . Such comparison shows that the annealed damage after implanting Ge ions at $0^{\circ} \mathrm{C}$ is much lower than that observed for B ions. Despite a high amount of damage is produced by

(a)

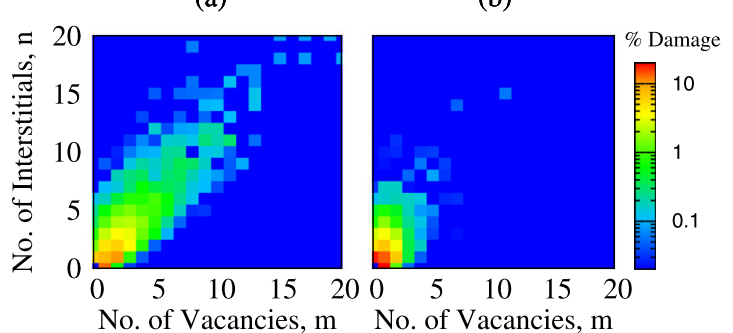

(c)

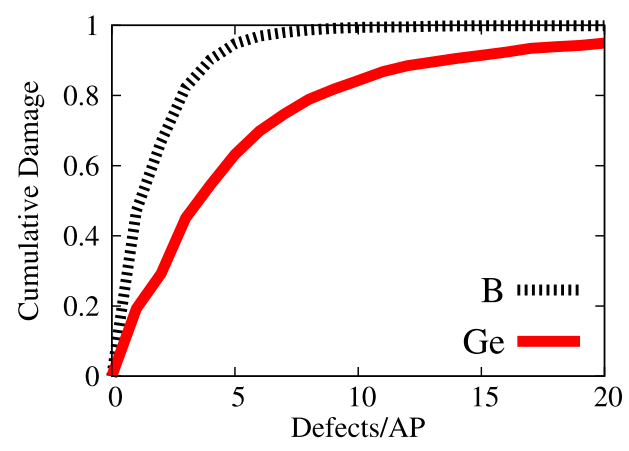

FIG. 4. 2D Concentration distribution histogram of $A P$ composition, $I_{n} V_{m}$, resulting after simulations of $150 \mathrm{keV} \mathrm{Ge} \mathrm{(a)} \mathrm{and} 40 \mathrm{keV} \mathrm{B} \mathrm{(b)} \mathrm{implants} \mathrm{at}$ $\mathrm{LN}_{2} \mathrm{~T}$. (c) The normalized cumulative damage distribution of the same data for both Ge (solid line) and B (dashed line) implantation as a function of the number of defects forming an $A P$. Fluences were chosen to generate the same amount of damage (Is and $V \mathrm{~s}$ ) for both conditions.

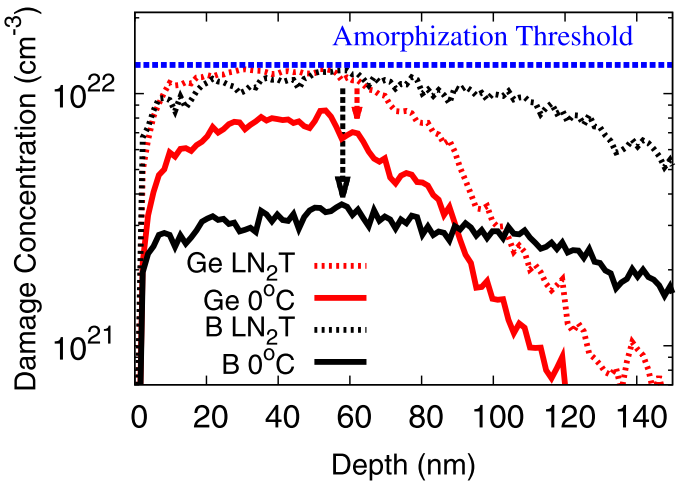

FIG. 5. Damage profiles caused by $150 \mathrm{keV} 2.25 \times 10^{13} \mathrm{Ge} / \mathrm{cm}^{2}$ (red lines) and $40 \mathrm{keV} 5 \times 10^{14} \mathrm{~B} / \mathrm{cm}^{2}$ implants (black lines) at both $\mathrm{LN}_{2} \mathrm{~T}$ and $0^{\circ} \mathrm{C}$. Vertical arrows show the quantity of dynamically annealed damage when comparing the cryogenic implantations with those performed at $0{ }^{\circ} \mathrm{C}$. The amorphization threshold is also represented (blue dashed line).

B ions at $\mathrm{LN}_{2} \mathrm{~T}$, it is readily annihilated at relatively low temperatures. This description is able to explain the narrowness of the $\alpha$-Ge layers reported for B implants. ${ }^{7}$ Such phenomena are described in our model by: (i) considering that light ions are more susceptible to suffer channeling events, which leads to less concentrated damage profiles than those for heavy ions, ${ }^{21}$ and (ii) the increasing recombination energies for increasing $A P$ sizes (Figs. 1 and 3), favoring recombinations for those small and diluted $A P$ s.

This model has been able to predict $\alpha / c$ interface locations for a wide range of conditions. Fig. 6 is a comparison between experimentally measured and predicted $\alpha / c$ interfaces for different experimental measurements found in the literature for $\mathrm{B},{ }^{7,8} \mathrm{~F},{ }^{14}$ and $\mathrm{Ge}^{4,7,14}$ ions. Other conditions, for which other mechanisms take place will be discussed in Sec. III D. Uncertainty in the measurements (when provided) is shown by bars in the y-axis, while error bars in the predicted results stand for simulated roughness. The consonance between these results and reported observations, in this work and in the literature, supports the followed approach. In cases for which neither channeling nor dynamic annealing take place, this and the CDED model (see Ref. 7) converge to

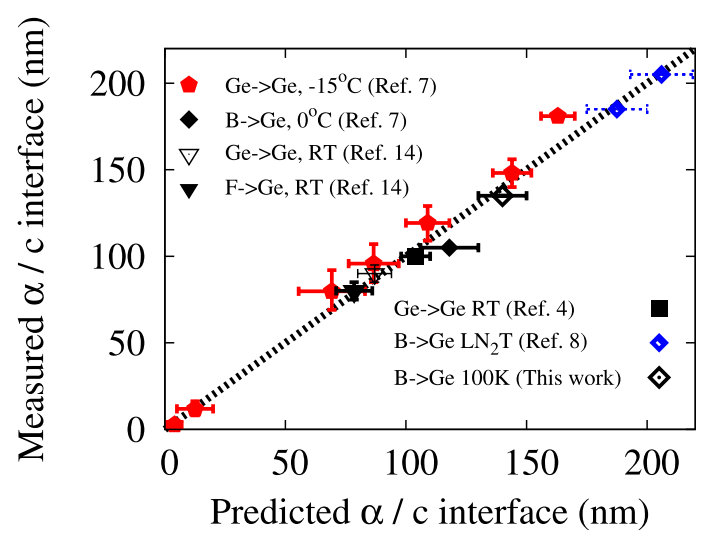

FIG. 6. Location of $\alpha / c$ interfaces measured experimentally (y-axis) for $150 \mathrm{keV} \mathrm{Ge}$ implantations at $-15^{\circ} \mathrm{C}$ at different fluences, ${ }^{7} 100 \mathrm{keV}$ $2 \times 10^{14} \mathrm{Ge} / \mathrm{cm}^{2}$ at RT, ${ }^{14} 35 \mathrm{keV} 1 \times 10^{15} \mathrm{~F} / \mathrm{cm}^{2}$ at RT, ${ }^{14} 100 \mathrm{keV} 1 \times 10^{15}$ $\mathrm{Ge} / \mathrm{cm}^{2}$ at $\mathrm{RT},{ }^{4} 40 \mathrm{keV} 5 \times 10^{15} \mathrm{~B} / \mathrm{cm}^{2}$ at $0{ }^{\circ} \mathrm{C},{ }^{7} 35 \mathrm{keV} \mathrm{B}$ at $\mathrm{LN}_{2} \mathrm{~T}$ for different fluences, ${ }^{8}$ and $40 \mathrm{keV} 1 \times 10^{15} \mathrm{~B} / \mathrm{cm}^{2}$ at $100 \mathrm{~K}$ of this work. Simulated $\alpha / c$ locations are represented in $\mathrm{X}$-axis. 
similar results, as the approach taken is qualitatively similar for such conditions (i.e., the damage is accumulated until it overcomes a certain threshold, for which the $c-\alpha$ relaxation occurs). Otherwise our results are in a very good agreement with different experiments reported in the literature, shown by the proximity of the points to the dashed line. Agreement between experimental results for B, F, and Ge implants and our simulations serves as a validation of the presented model and expands the predicting capabilities on Ge amorphization and damage generation.

\section{Cluster dissolution}

Results for damage accumulation towards amorphization have been presented so far for conditions in which only structural processes and/or dynamic annealing through $A P$ recombination are dominant. Nevertheless, for conditions of higher temperatures and/or low fluxes, we have investigated that other mechanisms as cluster dissolution can serve as an explanation for reported experiments. ${ }^{5,8,9,41}$

Fig. 7 shows a comparison between experimental, ${ }^{8}$ measured by means of c-RBS techniques, (symbols) and simulation results (lines) of the concentration per unit area of implantation of Is generated by $35 \mathrm{keV}$ B ions implanted at RT. With the model and calibration presented so far, agreement between experimental measurements and simulations could not be achieved (black dashed line). By investigating the damage left after those simulations, nearly only pure $I$ - and $V$-type stable clusters were present, evidencing that other physical mechanisms were needed to reproduce those results.

On the one hand, binding energies starting at $\sim 0.26 \mathrm{eV}$ were needed to activate $I$ and $V$ emissions in our simulations at these conditions. On the other hand, Sueoka et al. reported, by using $a b$ initio techniques, a binding energy for di-vacancies in Ge of $E_{b}\left(V_{2}\right)=0.58 \mathrm{eV}$ and a vacancy formation energy of $E_{f}(V)=2.56 \mathrm{eV}$. Di-vacancy formation energy for those results can be calculated as $E_{f}\left(V_{2}\right)=2 \times E_{f}(V)$ $-E_{b}\left(V_{2}\right)=4.54 \mathrm{eV}$ and $4.6 \mathrm{eV}$ for our parametrization (Table I). These results enabled us to identify the mechanisms starting to appear at these conditions.

Regarding binding and formation energies of the rest pure $V$ - or $I$-type clusters, we have performed a simple calibration in order to fit experimental results. Binding energies

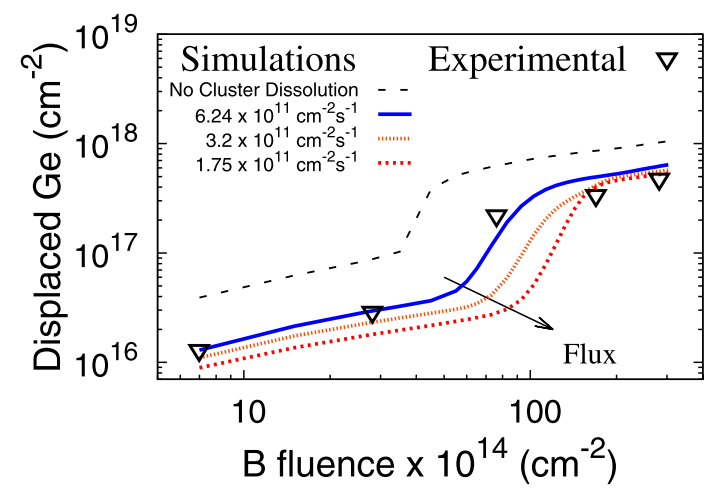

FIG. 7. Displaced Ge atoms for different $35 \mathrm{keV}$ B fluences at RT. Symbols represent experimental data. ${ }^{8}$ Simulation results (lines) for different fluxes are also presented along with results obtained without cluster dissolutions. are interpolated for both type of clusters from the mentioned $0.26 \mathrm{eV}$ for $E_{b,\{n, m\}=2}=0.26 \mathrm{eV}$, and up to $E_{b,\{n, m\}=50}$ $=1.2 \mathrm{eV}$. Even though a more systematic study on cluster binding and formation energies should be carried out in order to clearly identify these energies, (for example, by analyzing end of range defects, EOR, dissolution experiments ${ }^{4,9,41}$ ) this simple calibration has been able to achieve a good agreement between experimental results and our simulations.

Due to the number of mechanisms that start to take place at these conditions, and their complexity, knowledge on the flux is extremely important in order to be able to perform realistic simulations and identify the underlying processes. Conversion between current density, often expressed in $\mu \mathrm{A} / \mathrm{cm}^{2}$, and ion flux is not always straightforward and can even depend on the specific experimental setup. In Ref. 8, an average current density of $\sim 0.1 \mu \mathrm{A} / \mathrm{cm}^{2}$ is reported. To analyze the impact of changing dynamic annealing times at these conditions, we have performed simulations for a set of three different fluxes of B ions, which are presented in Fig. 7. It can be observed that an overall reasonable match for the amount of generated damage can be achieved for variable fluxes.

Comparing these results with those at $\mathrm{LN}_{2} \mathrm{~T}$ in Fig. 2, the drop in the damage concentration is again noticeable, specially at the first stages of the implantation. This shows, jointly with the studies of Impellizzeri and co-workers, ${ }^{8}$ the amount of damage that is annihilated at RT for B implantations in Ge. In the simulation results shown in Fig. 2, the three different regions of implantation regimes are clearly distinguished, and Region III is rapidly achieved. On the contrary, for implantations at RT (Fig. 7) the damage does not saturate completely to Region III for high fluences. This can be linked to the fact that no $\alpha$-Ge continuous layers were experimentally observed. Nevertheless, our model predicts formation of amorphizing areas, which is noticeable at the dramatic increase for the third experimental point. We have also observed in our simulations the formation of $\alpha$-Ge continuous layers for the highest reported fluence at RT. However, as it can be observed, even though Region III is not achieved, damage quantity is starting to saturate. This may serve as an indicator of that amorphization of the target might start for slightly higher fluences. Moreover, for the same quantity of damage in the $\mathrm{LN}_{2} \mathrm{~T}$ case, Region III is already starting, with a large continuous amorphous layer.

Fig. 8 is a comparison between experimental measurements ${ }^{5}$ (symbols) of relative disorder induced in the Ge sample by $30 \mathrm{keV} \mathrm{Ga}$ implantations for different fluences and ion fluxes at $\mathrm{RT}$ and $250^{\circ} \mathrm{C}$, and our simulations (lines). A very good agreement is found for nearly all the studied conditions. In order to compare these c-RBS measurements with our results, damage is normalized and saturated to 1 at values from which Region III is achieved for every condition. The comparison between all of these conditions, reported by Posselt et al. ${ }^{5}$ showed that amorphization behavior for Ga implants at RT does not depend strongly on the ions flux. On the contrary, while implanting at $250{ }^{\circ} \mathrm{C}$, amorphization could be achieved for an extremely high flux, while a negligible amount of damage is accumulated at low fluxes. As in the case for B implantations at RT (Fig. 7), the calibration for cluster dissolution upon the presented binding energies 


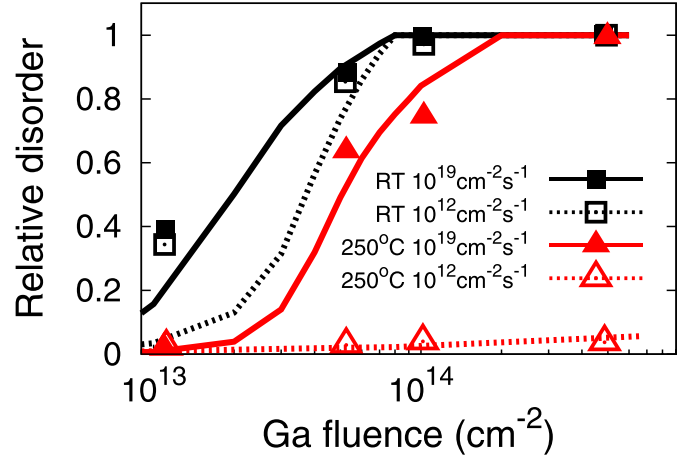

FIG. 8. Experimental measurements ${ }^{5}$ (symbols) and simulation results (lines) of the relative disorder induced in the Ge lattice after $30 \mathrm{keV} \mathrm{Ga}$ implantations at both RT and $250^{\circ} \mathrm{C}$ for both $10^{12} \mathrm{~cm}^{-2} \mathrm{~s}^{-1}$ and $10^{19} \mathrm{~cm}^{-2} \mathrm{~s}^{-1}$ fluxes.

was needed to not achieve amorphization in our simulations for the non-amorphizing flux at $250{ }^{\circ} \mathrm{C}$. Disagreement on the beginning of damage accumulation at RT can be explained as a consequence from a not exhaustive parametrization on cluster dissolution depending on their size. Despite this disagreement, the model predicts fairly enough the damage quantity and accumulation regimes up to amorphization for these very different conditions. Unfortunately, in the reported article, no information about the extension of the amorphous layers was given. Thus, these results are not included in Fig. 6.

Finally, we have noticed in our simulations that, while $V$-type cluster dissolution leads to a reduction in the number of these defects but an increase in their size, the case of I-type clusters is much different. With the presented model, a reduction in both number and their size is observed. This behavior has already been reported ${ }^{9,41}$ and the mechanism underlying this non-conservative cluster dissolution is still unknown. We attribute this behavior to the low diffusion rate of Is (Table I), making the emitted Is not able to travel far from the emitting cluster, while $V$ s are able to either recombine or grow other clusters. These explanations can serve as a starting point for a more specific cluster energetic study, and a hint for this non-conservative behavior.

Further work on the damage accumulation behavior for other heavy ions or noble gases, ${ }^{6}$ and deeper comparisons with other models for $\mathrm{Ge}^{10}$ and $\mathrm{Si}^{17}$ would increase the validity of existing models and understanding of damage accumulation and dissolution in Ge.

\section{CONCLUSIONS}

To summarize, we have presented a comprehensive model for $\mathrm{Ge}$ damage accumulation and amorphization caused by ion implantation. The model is based on an examination of the different stages of Ge amorphization that take place within the implantation process.

The main mechanisms analyzed in this work are summarized as follows: (i) a threshold value of $\sim 1.3 \times 10^{22} \mathrm{~cm}^{-3}$ damage concentration, for which the $\alpha / c$ transition occurs, and explains different implantation regimes. This value is independent of the implantation conditions. (ii) Recombination energies of $A P$ s depend on their size. These energies span the range between $0.5 \mathrm{eV}$ and $2.17 \mathrm{eV}$. for $\mathrm{Ge}$ SPER. (iii)
Dissolution of pure $I$ - and $V$-type clusters, which play an important role for temperatures equal or higher than RT at high fluences.

This description is able to explain and predict experimental results on damage accumulation and amorphization of $\mathrm{Ge}$ for a wide range of conditions. The model has been validated by reproducing reported experiments of $\mathrm{B}, \mathrm{F}, \mathrm{Ga}$, and $\mathrm{Ge}$ ions under different temperatures, fluences, and fluxes of implantation. Due to the different migration barriers of $I \mathrm{~s}$ and $V \mathrm{~s}$, cluster dissolution results in a different behavior in the evolution of $I$ - and $V$-type clusters.

Conclusions achieved in this study are (i) there are different implantation regimes based on the crystallinity and pre-existing damage within the Ge sample; (ii) a correct calculation upon the atomistic composition of the generated damage becomes crucial for predicting $\alpha / c$ interface locations; (iii) the diluted implantation-induced damage by light ions is easily annealed dynamically in Ge at relatively low temperatures; (iv) induced damage by heavier ions is more compact and consists of a higher number of $I \mathrm{~s}$ and $V \mathrm{~s}$; and (v) cluster dissolution becomes important for temperatures equal or higher than RT at high fluences and/or low fluxes.

\section{ACKNOWLEDGMENTS}

J.L.G.-S. acknowledges L. Agudo-Merida (IMDEA Materials Institute) and R. Gonzalez-Arrabal (NFI-UPM) for enlightening discussions. IMDEA Materials Institute acknowledges funding by GLOBALFOUNDRIES. I.M.-B. acknowledges funding from the Spanish Ministry of Economy and Competitiveness (MINECO) under Ramon y Cajal fellowship RYC-2012-10639.

${ }^{1}$ R. Pillarisetty, Nature 479, 324 (2011).

${ }^{2}$ P. S. Goley and M. K. Hudait, Materials 7, 2301 (2014).

${ }^{3}$ K. J. Kuhn, IEEE Trans. Electron Devices 59, 1813 (2012).

${ }^{4}$ A. Satta, E. Simoen, T. Clarysse, T. Janssens, A. Benedetti, B. De Jaeger, M. Meuris, and W. Vandervorst, Appl. Phys. Lett. 87, 172109 (2005).

${ }^{5}$ M. Posselt, L. Bischoff, D. Grambole, and F. Herrmann, Appl. Phys. Lett. 89, 151918 (2006).

${ }^{6}$ S. Decoster and A. Vantomme, J. Phys. D: Appl. Phys. 42, 165404 (2009).

${ }^{7}$ S. Koffel, P. Scheiblin, A. Claverie, and G. Benassayag, J. Appl. Phys. 105, 013528 (2009).

${ }^{8}$ G. Impellizzeri, S. Mirabella, E. Bruno, A. M. Piro, and M. G. Grimaldi, J. Appl. Phys. 105, 063533 (2009).

${ }^{9}$ A. Claverie, S. Koffel, N. Cherkashin, G. Benassayag, and P. Scheiblin, Thin Solid Films 518, 2307 (2010).

${ }^{10}$ G. Impellizzeri, S. Mirabella, and M. G. Grimaldi, Appl. Phys. A 103, 323 (2011).

${ }^{11}$ S. Mirabella, D. De Salvador, E. Napolitani, E. Bruno, and F. Priolo, J. Appl. Phys. 113, 031101 (2013).

${ }^{12}$ Y.-L. Chao, S. Prussin, J. C. S. Woo, and R. Scholz, Appl. Phys. Lett. 87, 142102 (2005).

${ }^{13}$ A. Claverie, C. Vieu, J. Fauré, and J. Beauvillain, J. Appl. Phys. 64, 4415 (1988).

${ }^{14}$ S. Boninelli, G. Impellizzeri, F. Priolo, E. Napolitani, and C. Spinella, Nucl. Instrum. Methods Phys. Res. B 282, 21 (2012).

${ }^{15}$ I. Martin-Bragado, M. Jaraiz, P. Castrillo, R. Pinacho, J. E. Rubio, and J. Barbolla, Appl. Phys. Lett. 84, 4962 (2004).

${ }^{16} \mathrm{R}$. Duffy, M. Shayesteh, I. Kazadojev, and R. Yu, in 13th International Workshop on Junction Technology (IWJT) (2013), p. 16.

${ }^{17}$ K. R. C. Mok, F. Benistant, M. Jaraiz, J. E. Rubio, P. Castrillo, R. Pinacho, and M. P. Srinivasan, J. Appl. Phys. 103, 014911 (2008).

${ }^{18}$ J. F. Ziegler, J. P. Biersack, and M. D. Ziegler, SRIM, the Stopping and Range of Ions in Matter (SRIM Co., 2008). 
${ }^{19}$ R. E. Stoller, M. B. Toloczko, G. S. Was, A. G. Certain, S. Dwaraknath, and F. A. Garner, Nucl. Instrum. Methods Phys. Res. B 310, 75 (2013).

${ }^{20}$ R. Wittmann and S. Selberherr, Solid-State Electron. 51, 982 (2007).

${ }^{21}$ B. N. Guo, N. Variam, U. Jeong, S. Mehta, M. Posselt, and A. Lebedev, in Proceedings of the 14th International Conference on Ion Implantation Technology (IEEE, 2002), p. 131.

${ }^{22}$ Sentaurus Process User Guide, I-2013.12, Synpsys Inc.

${ }^{23}$ EWJ. Mitchell, Br. J. Appl. Phys. 8, 179 (1957).

${ }^{24}$ P. N. Keating, Phys. Rev. 145, 637-645 (1966).

${ }^{25}$ A. M. Mazzone and M. Servidori, Philos. Mag. Lett. 57, 85 (1988).

${ }^{26}$ I. Martin-Bragado, A. Rivera, G. Valles, J. L. Gomez-Selles, and M. J. Caturla, Comput. Phys. Commun. 184, 2703 (2013).

${ }^{27}$ See http://materials.imdea.org/MMonCa for MMonCa.

${ }^{28}$ L. Pelaz, L. A. Marques, and J. Barbolla, J. Appl. Phys. 96, 5947 (2004).

${ }^{29}$ A. Claverie, C. Vieu, J. Faure, and J. Beauvillain, Mater. Sci. Eng., B 2, 99 (1989).

${ }^{30} \mathrm{C}$. Vieu, A. Claverie, J. Faure, and J. Beauvillain, in Phase Transformation Kinetics in Thin Films (1992).
${ }^{31}$ A. Chroneos and H. Bracht, Appl. Phys. Rev. 1, 011301 (2014).

${ }^{32}$ J. Vanhellemont, P. Śpiewak, and K. Sueoka, J. Appl. Phys. 101, 036103 (2007).

${ }^{33}$ P. Śpiewak, J. Vanhellemont, K. Sueoka, K. J. Kurzydowski, and I. Romandic, J. Appl. Phys. 103, 086103 (2008).

${ }^{34}$ K. Sueoka and J. Vanhellemont, Mater. Sci. Semicond. Process. 9, 494 (2006).

${ }^{35}$ G. L. Olson and J. A. Roth, Mater. Sci. Rep. 3, 1 (1988).

${ }^{36}$ I. Martin-Bragado and V. Moroz, Appl. Phys. Lett. 95, 123123 (2009).

${ }^{37}$ G. Q. Lu, E. Nygren, M. J. Aziz, D. Turnbull, and C. W. White, Appl. Phys. Lett. 56, 137 (1990).

${ }^{38}$ B. L. Darby, B. R. Yates, I. Martin-Bragado, J. L. Gomez-Selles, R. G. Elliman, and K. S. Jones, J. Appl. Phys. 113, 033505 (2013).

${ }^{39}$ F. F. Morehead and B. L. Crowder, Radiat. Effects 6, 27 (1970).

${ }^{40}$ E. Napolitani, E. Bruno, G. Bisognin, M. Mastromatteo, D. De Salvador, G. G. Scapellato, S. Boninelli, F. Priolo, V. Privitera, and A. Carnera, Phys. Status Solidi A 211, 118 (2014).

${ }^{41}$ S. Boninelli, G. Impellizzeri, A. Alberti, F. Priolo, F. Cristiano, and C. Spinella, Appl. Phys. Lett. 101, 162103 (2012). 